\title{
Knowledge, Attitude and Acceptance of COVID-19 Vaccine among General Population in South India
}

\author{
Binu Mathew*, Dhanya George, Sunitt Thomas, Doddaya H \\ Department of Pharmacy Practice, NET Pharmacy College, Raichur, Karnataka, INDIA.
}

\begin{abstract}
Background: COVID-19 pandemic has affected globally causing loss of life and livelihood. Several vaccines have been approved against coronavirus disease and distributed in different regions. However, general community knowledge, attitude and acceptance towards COVID-19 vaccinations are poorly understood. Thus, the study aimed to investigate community knowledge, attitude and acceptance towards COVID-19 vaccinations in South India. Objectives: The objectives of the study were to assess the knowledge, attitude and acceptance regarding COVID-19 vaccination and to improve and increase vaccination coverage and completeness. Results: A total of 742 participants were enrolled in this study. Most of the respondents (42.58\%) were aged between $18-30$ years and $32.88 \%$ were in the $30-60$ years group. Majority of them said they will get vaccinated as early as possible $(52.02 \%)$ and $20.75 \%$ will delay the vaccination. $93.36 \%$ participants think that vaccines should be available free of cost and $48.51 \%$ are not ready to afford the vaccine at their own expense if it is not provided free of cost by the government. $53.63 \%$ participants are concerned about the safety and efficacy. Conclusion: The most important factor for vaccine hesitancy is the occurrence of mild or serious adverse effects following immunization. The present study revealed inadequate knowledge and positive attitudes towards COVID-19 vaccinations. The findings suggest immediate health education programs and more accurate information should be distributed and advertised by respective health authorities.
\end{abstract}

Key words: Coronavirus, Hesitancy, Immunity, Misinformation, Myths, Vaccination.

\section{INTRODUCTION}

The Coronavirus disease, COVID-19, has been rapidly affecting millions of people over 144 countries. The pandemic became a significant threat to the public health system and also posed economic consequences globally. ${ }^{1}$ The efforts to lessen the effects of the pandemic, and to reduce the health and socio-economic impact relies to a large extent on the preventive methods. Thus, huge efforts by the scientific community and pharmaceutical industry backed by governments' support, were directed towards developing efficacious and safe vaccines for SARS-CoV-2. ${ }^{2}$

Vaccines are an important method for preventing COVID-19 pandemic from spreading. Given the vast population and the relatively high vaccine hesitancy for current vaccines as well as poor vaccination coverage, understanding vaccine acceptance is critical. Myths, rumors and misinformation can also spread quickly online, especially via social media.

Uncertainty regarding COVID-19, whether people have natural immunity and whether complex home remedies help protect against coronavirus, may have been exacerbated by reliance on social media. ${ }^{3}$ That may also explain why some people aren't sure whether the virus was created by humans and released on purpose. Uncertainty and rapidly evolving knowledge may have led to the virus's increased fear. Many lay media and websites have relentlessly reported on new cases and deaths in real time, sometimes
DOI: $10.5530 / \mathrm{ijopp} .15 .1 .7$

Address for correspondence: Mr. Binu Mathew,

Department of Pharmacy Practice, NET Pharmacy College, Raichur-584103, Karnataka, INDIA.

Email id: sunittthomas@gmail. com

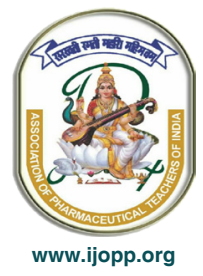


giving unauthorized medical advice without waiting for confirmation. The sheer volume and diversity of news has resulted in a huge information overload, resulting in a true infodemic. These results highlight the significance of disseminating reliable COVID-19 health information across a variety of channels in order to reach the general public and correct misinformation.

Although providing the public with evidence-based scientific data is helpful and important, it does not guarantee that people will understand or interpret the data correctly. If too much information is published, the public becomes flooded with different facts and inconsistent information, leading to emotional responses and distrust of decision-makers; evidence-based data runs the risk of being self-defeating. Furthermore, as seen in the current COVID-19 situations, debates among individuals and organizations with a large web and media presence often result in opposing opinions and negative beliefs. ${ }^{4}$

Vaccine hesitancy is described by the World Health Organization (WHO) Strategic Advisory Group of Experts on Immunization as a 'delay in acceptance or rejection of vaccination despite the availability of vaccination services, which may differ in type and severity depending on when and where it occurs, as well as the vaccine involved, as multiple studies have verified. Vaccine hesitancy is becoming a global concern in reality, WHO has named it one of the top ten global health threats for 2019. Vaccine apprehension and misinformation are significant barriers to achieving coverage and population immunity in many countries.

Governments, public health agencies and advocacy organizations must be ready to combat vaccine apprehension and increase vaccine awareness so that the public accepts immunization when it is necessary. Antivaccination groups are now protesting against the need for a vaccine in a number of nations, with some also denying that COVID-19 exists at all. Misinformation disseminated across multiple networks could have a significant impact on COVID-19 vaccine acceptance. The rapid speed of vaccine production has exacerbated public concerns and could jeopardize acceptance. ${ }^{5}$

Governments must be prepared to ensure equitable access to and distribution of COVID-19 vaccines on a large scale. This will include more capacity for the health system, as well as strategies to boost confidence in and awareness of the vaccine and those who administer it. Governments, public health departments and public advocacy groups must be prepared to address vaccine hesitancy and increase vaccine awareness in order for the community to embrace immunization where possible. Anti-vaccination advocates are already campaigning in many countries to eliminate the need for a vaccine, although others doubt COVID-19's existence altogether. Misinformation disseminated through various networks may have a significant impact on COVID-19 vaccine's approval. Governments and communities must determine current levels of readiness in order to obtain a COVID-19 vaccine that is potentially safe and effective, as well as identify reasons for scepticism and or vaccine approval. ${ }^{6}$

\section{OBJECTIVES OF THE STUDY}

The objectives of the study were to assess the knowledge, attitude and acceptance regarding COVID- 19 vaccination and to improve and increase vaccination coverage and completeness.

\section{METHODOLOGY}

This cross-sectional study was conducted for a period of three months from March 2021 to May 2021 among the general public of South India. The required data was collected through distribution of a validated pre designed questionnaire. All the collected data was subjected for evaluation.

\section{Inclusion Criteria}

All people above the age of 18 , who were willing to participate in the study were included.

\section{Exclusion Criteria}

People who were not willing to participate in the study were excluded from the study.

\section{Study tools}

The instrument used in the study was a two-part questionnaire. Section-1 deals with socio- demographic characteristics of respondents like education level and occupation. Section-2 deals with answers to the questions based on knowledge and attitude and acceptance regarding COVID- 19 vaccination. Questionnaires are defined as a set of printed or written questions with the choice of answers, devised for the purpose of a survey or statistical study. The validated pre designed questionnaire was taken from previous research articles.

\section{RESULTS}

A total of 742 participants were enrolled in this study. Socio demographic data of the participants are shown 
in Table 1. Most of the respondents (42.58\%) were aged between $18-30$ years and $32.88 \%$ were in the $30-60$ years group. Among these $51.48 \%$ were male participants and $48.51 \%$ were female participants. Most of the respondents were graduates $(69.54 \%)$, and $19.67 \%$ were having higher secondary levels of education and $6.46 \%$ were post graduates.

The response of participants regarding the knowledge of COVID-19 vaccine is good. Majority of the participants $(81.40 \%)$ agreed that vaccination is a simple, safe and effective way of protecting people against harmful diseases, before they come into contact with them. $43.12 \%$ participants said we should get vaccinated to protect ourselves whereas $54.98 \%$ participants said we should get vaccinated to protect ourselves as well as others. Most of the participants (97.30\%) heard of COVID-19 vaccine. Majority of the respondents (51.21\%) got information about COVID-19 vaccine through news media and $27.76 \%$ got it through official media.77.89\% of the participants were aware that they should wear mask even after vaccination and majority of them knew that there is possibility of infection even after vaccination.

Majority of participants (43.12 percentage) are concerned about the long term safety and efficacy of the vaccine as shown in Table 2. Factors affecting the decision to be vaccinated are given in Table 3. Preventive measures used by participants other than the vaccine are given in Figure 1.

A major proportion of the respondents had a positive attitude towards vaccination (54.71\%) and 51.21\% said they would encourage their friends and family to take vaccination. Majority of them said they will get

Table 1: Demographics and social data ( $n=742)$.

\begin{tabular}{lcc}
\multicolumn{1}{c}{ Variables } & $\begin{array}{c}\text { Number of } \\
\text { participants }\end{array}$ & $\begin{array}{c}\text { Percentage } \\
\text { (\%) }\end{array}$ \\
\hline 1. Age distribution (in years) & & \\
18- 30 & 316 & 42.58 \\
$30-60$ & 244 & 32.88 \\
Above 60 & 182 & 24.52 \\
2. Gender & & \\
Male & 382 & 51.48 \\
Female & 360 & 48.51 \\
3. Education & & \\
Primary & 32 & 4.31 \\
Higher secondary & 146 & 19.67 \\
Graduate & 516 & 69.54 \\
Post Graduate & 48 & 6.46 \\
\hline
\end{tabular}

\begin{tabular}{|c|c|c|c|}
\hline SI.no & $\begin{array}{c}\text { Concerns about } \\
\text { COVID-19 } \\
\text { vaccine }\end{array}$ & $\begin{array}{c}\text { Number of } \\
\text { participants }\end{array}$ & $\begin{array}{c}\text { Percentage } \\
(\%)\end{array}$ \\
\hline 1. & $\begin{array}{l}\text { Long term safety } \\
\text { and efficacy }\end{array}$ & 320 & $43.12 \%$ \\
\hline 2. & Side-effects & 270 & $36.38 \%$ \\
\hline 3. & $\begin{array}{l}\text { Don't believe it } \\
\text { will be solution for } \\
\text { COVID-19 }\end{array}$ & 44 & $5.92 \%$ \\
\hline 4. & $\begin{array}{l}\text { I believe it will } \\
\text { have negative } \\
\text { effects on health }\end{array}$ & 44 & $5.92 \%$ \\
\hline 5. & Others & 64 & $8.62 \%$ \\
\hline
\end{tabular}

Table 3: Factors which would help if not yet decided to be vaccinated ( $n=742)$.

\begin{tabular}{cccc} 
SI.no. & $\begin{array}{c}\text { Which of the } \\
\text { following would help } \\
\text { if not yet decided to } \\
\text { be vaccinated }\end{array}$ & $\begin{array}{c}\text { No. of } \\
\text { participants }\end{array}$ & $\begin{array}{c}\text { Percentage } \\
\text { (\%) }\end{array}$ \\
\hline $1 . \quad$ If my doctor approves & 254 & $34.23 \%$ \\
2. I want to wait for a & 300 & $40.43 \%$ \\
period to observe how \\
other people react \\
If detailed written \\
review is shared with \\
public on how the \\
vaccine is produced \\
and what it contains \\
$\quad$ If legal authorities \\
would approve
\end{tabular}

vaccinated as early as possible (52.02\%) and $20.75 \%$ will delay the vaccination. But $37.19 \%$ of respondents had a neutral attitude towards vaccination and $25.33 \%$ of them will suggest their friends and family to delay the vaccination which shows the lack of awareness about the vaccination. $50.94 \%$ of participants believe that the vaccine is having side-effects. 236 participants (31.80\%) believe that COVID-19 may be eradicated without vaccination, if everyone in the society maintains the preventive measures. $57.68 \%$ participants think that everyone should get vaccinated, $54.44 \%$ believe that healthcare workers are supposed to get vaccinated first and $27.49 \%$ thought it to be senior citizens. Majority of participants $55.25 \%$ believe that people with a history of severe allergic reactions to any ingredients of vaccine, those who are currently sick or experiencing symptoms of COVID-19 and pregnant or lactating women should be excluded from receiving vaccine to avoid possible adverse effects. $93.36 \%$ participants think that vaccines should be available free of cost and $48.51 \%$ are not 
Preventive measures used apart from COVID-19 vaccine

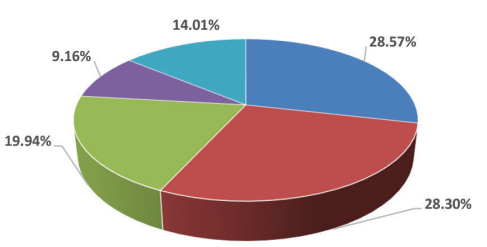

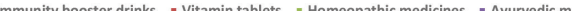

Figure 1: Preventive measures used apart from COVID-19 vaccine $(n=742)$.

ready to afford the vaccine at their own expense if it is not provided free of cost by the government. 53.63\% participants are concerned about the safety and efficacy of vaccines whereas $35.57 \%$ needed more information on vaccine safety and efficacy. 5.92\% do not believe vaccines as a solution for COVID-19. 40.43\% of participants prefer to wait for a certain period to observe how other people react to the vaccine and $34.23 \%$ said they would get vaccinated if their doctor gives approval. 55.25\% believe that a prepared script on the importance of getting the vaccine with an explanation on its safety may help improve the attitude of people who are resistant to get the vaccine. Majority participants $76.81 \%$ were unaware and $8.89 \%$ think it's not necessary to selfquarantine, if exposed to someone with COVID-19 after fully being vaccinated. $53.36 \%$ did not know the time interval between vaccine doses.

\section{DISCUSSION}

Our study is focused only in southern states of India. It has been observed that more than half of the respondents were ready to get vaccinated if offered and would also encourage others to get vaccinated. Even though more than $50 \%$ of the participants have a positive attitude towards COVID-19 vaccine, there are still confusions prevailing regarding the vaccine. People are concerned about safety and efficacy of the vaccine as it is made within a short period of time and hence some of them need a certain time period to observe others reaction and health conditions after vaccination. Participants also had doubts like how they can rely on the vaccine if the people can still get infected even after being vaccinated.

We have enquired about vaccine affordability and it was observed that the majority of the participant's demands free vaccines from the government.

Active participation of healthcare workers in promoting the need to get vaccinated can improve vaccine acceptance among the public. Government should also ensure their participation in improving people's attitude towards the vaccine. Vaccine hesitancy or ignorance should not be encouraged. The misconceptions regarding the vaccine can be removed by providing necessary awareness programs. Governments can play their role in conducting various awareness programs.

\section{CONCLUSION}

The most important factor for vaccine hesitancy is the occurrence of mild or serious adverse effects following immunization. The present study revealed inadequate knowledge and positive attitudes towards COVID-19 vaccinations. Study reveals that the public are interested in free vaccines from the government. The findings suggest immediate health education programs and more accurate information should be distributed and advertised by respective health authorities.

\section{ACKNOWLEDGEMENT}

Authors take it as a privilege to acknowledge Sri S R Reddy; Chairman Navodaya Educational Trust, Principal, HODs and Staff of NET Pharmacy College for their support during the study.

\section{CONFLICT OF INTEREST}

The authors declare no conflict of interest.

\section{ABBREVIATIONS}

COVID - 19: Coronavirus disease 2019.

\section{REFERENCES}

1. Al-Mohaithef M, Padhi BK. Determinants of COVID-19 vaccine acceptance in Saudi Arabia: a web-based national survey. J Multidiscip Healthc. 2020;13:1657-63. doi: 10.2147/JMDH.S276771, PMID 33262600.

2. Sallam M. COVID-19 vaccine hesitancy worldwide: a concise systematic review of vaccine acceptance rates. Vaccines. 2021 Feb;9(2):160. doi: 10.3390/ vaccines9020160, PMID 33669441

3. Mannan DK, Farhana KM. Knowledge, attitude and acceptance of a COVID-19 vaccine: A global cross-sectional study. Int Res J Bus. Soc Sci. 2020 Dec 7;6(4)

4. Biasio LR, Bonaccorsi G, Lorini C, Pecorelli S. Assessing COVID-19 vaccine literacy: a preliminary online survey. Hum Vaccin Immunother. 2021 May 4;17(5):1304-12. doi: 10.1080/21645515.2020.1829315, PMID 33118868.

5. Lazarus JV, Ratzan SC, Palayew A, Gostin LO, Larson HJ, Rabin K, Kimball S, El-Mohandes A. A global survey of potential acceptance of a COVID-19 vaccine. Nat Med. 2021 Feb;27(2):225-8. doi: 10.1038/s41591-020-1124-9, PMID 33082575.

6. Gautam A, Dhara B, Mukherjee D, Mukhopadhyay D, Roy S, Ganguly SS, et al. A digital survey on the acceptance and affordability of COVID-19 vaccine among the people of West Bengal, India-A survey based study. medRxiv. 2020 Jan 1. 